\title{
La radioprotection : exemple d'action contre les nuisances du monde moderne
}

Allocution prononcée lors de l'ouverture du $4^{e}$ Congrés International de I'Association Internationale de Radioprotection. tenu à Paris du 24 au 30 avril 1977. par le

\author{
Dr. H. P. JAMMET (*). \\ président du Congrès.
}

L'historique de la radioprotection montre que cette discipline est passée par trois phases sucessives au cours des trois premiers quarts de ce siècle : la première a été celle de la découverte des nuisances radiologiques et radioactives; la seconde a été celle de l'organisation de la détection et de la prévention contre les nuisances radiologiques dans le domaine professionnel; la troisième a été celle de la mise sur pied d'un ensemble cohérent de protection contre les nuisances liées à l'énergie nucléaire aussi bien dans le domaine public que dans le domaine professionnel.

Si aujourd'hui, la radioprotection peut être considérée comme un exemple d'action contre les nuisances du monde moderne, c'est en raison de ses réalisations, de son caractère multidisciplinaire et de son apport à la protection en général.

Il est couramment admis dans l'opinion publique, et malheureusement dans certains cercles scientifiques mal informés, que les nuisances radiologiques et radioactives sont mal connues. Or, la radioprotection peut se prévaloir du contraire et elle constitue en ce domaine un exemple. En effet, qu'il s'agisse de rayonnements électromagnétiques ou particulaires, le spectre est bien défini. Sur le plan des nuisances radioactives, on peut dire que la totalité des radio-éléments nocifs est parfaitement connue, qu'il s'agisse de leur structure propre, de leur schéma de désintégration ou de leur période radioactive. Un effort considérable a été fait, en outre, sur la forme physicochimique des supports matériels de la radioactivité, qu'il s'agisse de solutions, de suspensions, d'aérosols ou de poussières. Si l'on considère le domaine si complexe des nuisances rencontrées

(*) Commissariat à l'Énergie Atomique, Institut de Protection et de Sûreté Nucléaire. Département de Protection, B.P. $\mathrm{n}^{*}$ 6, 92260 Fontenay-aux-Roses.

RADIOPROTECTION, VOL. $13-\mathrm{N}^{\circ} 1$ 
dans les mines d'uranium, connaît-on un autre exemple de contamination atmosphérique pour lequel on possède autant de précisions sur la granulométrie, la forme physicochimique, les caractéristiques et les équilibres radioactifs ?

La mesure des nuisances radiologiques a commencé il y a bien longtemps par des méthodes empiriques dont certaines consistaient à utiliser leur action biologique nocive. Mais la radioprotection a su profiter du développement de la physique et de l'électronique pour maîtriser d'une façon remarquable la mesure des rayonnements. Il est peu de domaines dans lesquels la précision de l'estimation des nuisances soit aussi grande que pour les rayonnements électromagnétiques et ceci aussi bien par la mesure des champs de rayonnement que par celle de l'irradiation individuelle des personnes exposées. Pour les rayonnements particulaires, on se heurte à de grandes difficultés techniques, par exemple dans la mesure des rayonnements neutroniques. Cependant, les efforts persévérants sont couronnés peu à peu de succès. Mais c'est dans le domaine de l'analyse des nuisances radioactives que la radioprotection peut être citée comme un exemple. En effet, est-il possible d'envisager une précision et une sensibilité aussi grande que celles qui permettent de mesurer les nuisances radioactives à des niveaux qui sont de l'ordre du picocurie?

C'est également un lieu commun que d'entendre dire que les effets des radiations ionisantes sont très spéciaux et qu'ils sont mystérieux. Nombre de scientifiques, étrangers aux disciplines que sont la radiobiologie et la radiopathologie, se complaisent à émettre des idées parfaitement erronées. En effet, il peut paraître paradoxal que les nuisances radiologiques et radioactives apparues au $\mathrm{xx}^{e}$ siècle aient des effets mieux connus que ceux des nuisances physiques anciennes telles que la chaleur ou le bruit et que ceux de la plupart des nuisances chimiques ou biologiques. Mais c'est un des domaines oủ la radioprotection peut vraiment être citée en exemple. En effet, elle a su profiter du développement considérable de la radiobiologie et de la radiopathologie et intégrer pour ses besoins propres toutes les données scientifiques accumulées depuis 50 ans. Les radiations ionisantes lorsqu'elles agissent sur la matière vivante ne présentent pas de caractère de spécificité du fait de la loi biologique générale qui veut que les cellules, les tissus et les organes répondent, selon des modalités qui leur sont propres, aux différentes agressions qu'ils peuvent subir. On comprend de ce fait que l'action des rayonnements ionisants ne puisse être ni spécifique ni mystérieuse et que les dommages consécutifs soient analogues et comparables à ceux d'autres agents nocifs. Mais là où apparaît vraiment la différence quand on fait la comparaison, c'est que pour les nuisances radiologiques et radioactives la connaissance des mécanismes d'une part, le bilan des actions nocives d'autre part, sont beaucoup mieux connus. Si nous considérons l'immense domaine des dommages liés d'une façon déterminante à l'action des nuisances radiologiques et radioactives, nous découvrons une précision remarquable dans la corrélation quantitative. Existe-t-il pour les brûlures thermiques ou chimiques des corrélations comparables à celles qui sont connues pour les brûlures radiologiques? Existe-t-il pour les effets hématologiques des agents chimiques ou biologiques des corrélations comparables à celles établies pour les radiations ionisantes? Existe-t-il pour les 
effets tératologiques des toxiques des corrélations comparables? On pourrait continuer ces exemples pour pratiquement tous les tissus et organes. Si nous considérons, par ailleurs, les dommages corrélés de façon stochastique aux agents nocifs, on constate que la radioprotection dispose d'un éventail de données incomparablement plus étendu et plus précis que pour tout autre domaine de protection. Est-il dans le domaine de la cancérogenèse, chimique ou biologique, des données aussi précises que celles de la cancérogenèse radiologique ou radioactive ? Ceci est tellement vrai qu'il n'est même pas nécessaire, pour établir les corrélations, de passer par l'expérimentation animale et l'extrapolation humaine subséquente. On dispose, par l'épidémiologie humaine, de données déjà largement suffisantes. Dans beaucoup de cas, on a pu établir de façon satisfaisante les corrélations dose-effet lorsqu'il s'agit de doses relativement élevées. Le domaine sur lequel portent les discussions est celui des faibles doses. Encore est-on capable de fixer l'enveloppe supérieure des dommages éventuels. Mais que peut-on dire de la plupart des autres nuisances pour lesquelles on ne connaît même pas les corrélations à forte dose ?

Enfin, dans le domaine des dommages génétiques, il faut bien considérer que la radioprotection est là également à l'avant-garde. L'expérimentation animale a fourni des données tout à fait valables en radiogénétique. Quel est le domaine de l'hygiène où l'on se préoccupe des dommages génétiques pour les populations? C'est seulement pour les rayonnements ionisants que ces problèmes ont été abordés. Aussi, apparait-il paradoxal que la radioprotection qui a fait cet effort d'avant-garde soit indirectement responsable de la pénalisation des activités impliquant les rayonnements ionisants. De tels effets génétiques existent certainement, et combien plus importants pour d'autres nuisances qui laissent indifférente l'opinion publique pour la seule raison qu'on ne s'est jamais préoccupé de leur existence.

Dans le domaine de la prévention, la radioprotection peut également être citée en exemple. En effet, les méthodes de protection ont été adaptées à des principes rigoureux de prévention qui s'efforcent d'obtenir que les rayonnements parasites et la pollution radioactive soient réduits à zéro ou à des fuites insignifiantes, La radioprotection a utilisé, à ses fins propres, tous les développements technologiques permettant de parvenir à ce but. Pour se mettre à l'abri des nuisances radiologiques dues aux rayonnements électromagnétiques et particulaires, des systèmes d'écrans plus ou moins complexes ont été mis en place, dont on trouve peu d'équivalents dans le domaine des nuisances thermiques, lumineuses ou acoustiques. Mais poussant plus loin, la radioprotection a fait un effort considérable dans le domaine de la télémanipulation et de l'automatisation, de façon à permettre que les opérations soient effectuées sans que les hommes soient exposés. De même, en ce qui concerne la contamination radioactive, la radioprotection est allée bien au-delà de ce que les chimistes avaient l'habitude de faire pour la manipulation de substances toxiques. Elle ne s'est pas contentée de simples hottes plus ou moins sophistiquées, mais a carrément envisagé l'utilisation de confinements hermétiques permettant d'effectuer des travaux chimiques ou métallurgiques à l'abri de toute pollution. 
L'industrie atomique et l'utilisation des radio-éléments posent obligatoirement des problèmes de déchets, d'effluents et de transport avec consécutivement des problèmes de pollution de l'environnement et d'impact écologique. Dans ce domaine également, la radioprotection peut être citée en exemple. En effet, en ce qui concerne l'éventuelle atteinte des systèmes écologiques, des travaux considérables ont été effectués dans le but de la radioprotection; des données relatives aux corrélations dose-effet ont été ainsi collectées. C'est ainsi qu'il a puêtre prouvé que dans l'échelle biologique, l'homme se trouve à une place qui le rend particulièrement sensible aux radiations. De ce fait, les précautions prises pour lui entraînent ipso facto une protection de la nature. Néanmoins, et compte tenu des accumulations ponctuelles qui peuvent se produire, des travaux importants continuent en ce domaine. Peut-on citer d'autres nuisances chimiques ou physiques pour lesquelles un effort aussi marqué ait été fait ? A ce sujet, il est intéressant de remarquer que les problèmes des effets écologiques de la pollution thermique ont pris une soudaine importance lorsqu'ils ont été rapportés à l'énergie nucléaire, alors que depuis longtemps ils existaient pour d'autres procédés de production d'énergie. C'est encore le mérite de la radioprotection de les avoir franchement abordés et d'y avoir déjà apporté des solutions. Mais ces effets de la pollution radioactive dans le domaine écologique ne peuvent s'analyser de façon satisfaisante que par une connaissance approfondie des transferts des polluants radioactifs dans le milieu. En fait, il s'agit d'étudier les modalités d'atteinte de l'homme à partir des sources de polluants radioactifs. Il faut reconnaître que la radioprotection a vu ses études facilitées du fait de la possibilité de détecter les substances radioactives à des niveaux extrêmement bas et par conséquent de les suivre à la trace. Il en résulte qu'actuellement le cheminement de la pollution radioactive au travers des vecteurs primaires que sont l'air et l'eau, des vecteurs intermédiaires que sont le sol, la flore et la faune, des vecteurs terminaux que sont les différentes composantes de la chaîne alimentaire est amplement connu. On a pu déterminer ainsi les flux au travers des différents maillons des chaines de transfert, les zones de dépôt, les cas de concentration ou de dilution relative. L'accumulation des données a été telle que des modèles de dispersion, de diffusion, de cheminement. ont pu être établis. Si l'on considère la pollution radioactive due aux expérimentations des armes nucléaires, on peut dire que la radioprotection a su tirer profit des données innombrables qui ont été obtenues à l'échelle régionale ou mondiale sur les transferts atmosphériques, océaniques, écologiques et alimentaires. Pour la pollution liée à l'industrie nucléaire, on a pu préciser l'impact des pollutions de caractère local, régional ou mondial. Peut-on, dans le domaine de la pollution chimique, citer des efforts comparables qui aient été faits, à part quelques exceptions ? C'est tout à l'honneur de la radioprotection d'avoir réalisé ces études non pour quelques polluants radioactifs privilégiés mais pour l'ensemble de tous ceux qui représentaient une nuisance significative.

S'il est un domaine dans lequel la radioprotection puisse être cité comme exemple d'action contre les nuisances du monde moderne, c'est bien celui de la réglementation et de la normalisation. En effet, il est parfaitement admis par tout le monde que la lutte contre les nuisances radiologiques et radioactives profite depuis un demi-siècle de l'existence d'une Commission Internationale de Protec- 
tion Radiologique (C.I.P.R.) ayant pour but d'élaborer des recommandations générales en matière de radioprotection. Pour aucun autre agent nocif, qu'il soit physique, chimique ou biologique, une telle situation n'existe et les spécialistes qui s'occupent des autres nuisances nous envient une telle organisation. Le résultat de cette situation est que, pour la radioprotection, les réglementations existant dans tous les pays du monde et adoptées par toutes les organisations internationales compétentes, sont fondées sur les mêmes principes. La C.I.P.R., à ses débuts, s'est trouvée en présence d'une situation alarmante due aux nombreux dommages corporels atteignant les travailleurs utilisant les rayonnements ionisants. Elle a donc établi des limites d'exposition dans le but de mettre ces travailleurs à l'abri. Au fur et à mesure du développement des connaissances en radiobiologie et en radiopathologie, elle a été amenée à modifier ces limites, en vue de tenir compte des dernières données disponibles. On a, peu à peu. abouti ainsi ả un système cohérent de limites à ne pas dépasser pour tous les types de rayonnements ionisants et la quasi-totalité des radionucléides incriminés. Ce système de limite d'exposition à lui seul constitue un exemple unique de réglementation. Mais devant l'extension de l'énergie nucléaire et de l'utilisation des substances radioactives, la C.I.P.R. a fait un effort doctrinal sans précédent dans le sens de la continuité et de l'approfondissement de ses principes. On est ainsi parvenu, d'une part à la justification des activités impliquant les nuisances radiologiques et radioactives, d'autre part à l'optimisation de la radioprotection pour obtenir les résultats les plus efficaces, tout en tenant compte des considérations économiques et sociales. La radioprotection peut donc s'enorgueillir de disposer actuellement de la doctrine la plus avancée qui ait été élaborée en la matière soit dans le domaine professionnel, soit dans le domaine public et qui, par ailleurs, a reçu la consécration de sa valeur pratique.

En effet, l'efficacité de la radioprotection a pu être démontrée grâce aux efforts des organisations nationales et internationales et en particulier du Comité Scientifique pour l'Étude des Effets des Rayonnements Ionisants, créé par l'Assemblée générale des Nations Unies en 1955. Ce Comité comme nul ne l'ignore, avait été créé pour analyser la pollution radioactive due aux expérimentations d'armes nucléaires et ses conséquences éventuelles. Il a vu son domaine s'étendre tout naturellement à l'ensemble des expositions radiologiques et radioactives auxquelles est soumise l'humanité. Il a effectué un travail de synthèse dont il n'existe aucun autre exemple pour quelque nuisance que ce soit. Ce travail comprend deux volets : l'un relatif au bilan des expositions, l'autre aux effets des rayonnements ionisants. Le bilan des expositions se présente actuellement comme remarquablement complet puisqu'il envisage l'irradiation naturelle, l'irradiation générale due à l'habitat et à l'alimentation, l'irradiation industrielle liée à l'énergie nucléaire et à l'utilisation des substances radioactives. l'irradiation liée aux expérimentations d'armes nucléaires, l'irradiation professionnelle et l'irradiation médicale liée à l'utilisation diagnostique et thérapeutique des rayonnements ionisants. La radioprotection est donc un exemple unique pour lequel on possède le bilan complet et précis de l'ensemble des expositions naturelles et artificielles auxquelles est soumise l'espèce humaine. Ces données sont complétées par une synthèse de toutes les connaissances actuellement disponibles en matière de radiobiologie et de radiopathologie 
permettant de connaître les mécanismes d'action des radiations et les corrélations entre les expositions et les dommages. Cet ensemble de données couvre aussi bien les effets immédiats que les effets tardifs cancérigènes et mutagènes. Comme on est loin de l'opinion couramment admise du mystère de l'action des radiations et de l'ignorance en cette matière ! Au contraire, la radioprotection peut être citée comme un modèle pour lequel les autorités internationales entretiennent l'effort de synthèse permanent qui permet de tenir à jour l'évolution des données scientifiques disponibles en la matière.

Enfin, bien que les accidents radiologiques et radioactifs soient peu fréquents, un effort considérable a été réalisé par la radioprotection pour les éviter et pour minimiser leurs conséquences. Les accidents radiologiques dus à des surexpositions entraînant des brûlures radiologiques localisées ne sont pas exceptionnels; ils font l'objet d'études très poussées et c'est peut-être un domaine où l'effort devrait être accentué. Les accidents radiologiques entrainant des irradiations généralisées sont, eux, exceptionnels et ont fait l'objet d'études très poussées tant sur le plan diagnostic que thérapeutique avec mise en œuvre des méthodes les plus sophistiquées de la médecine. Quant aux accidents nucléaires avec contamination significative des populations, il ne s'en est pratiquement pas produit. La radioprotection, là aussi, a su profiter des efforts de prévention dont l'ensemble constitue la sûreté radiologique des installations. Dans quel autre domaine d'activité un tel effort a-t-il été consenti en ce qui concerne la mise sur pied d'une sûreté exemplaire?

Comme il a déjà été dit, la radioprotection est un carrefour multidisciplinaire. C'est son mérite que d'avoir su intégrer des spécialistes venus d'horizons très divers: mathématicièns, physiciens, chimistes, ingénieurs, météorologistes, hydrologistes, géologues, agronomes, vétérinaires, écologistes, pharmaciens, médecins. Ceci aussi mérite d'être cité en exemple. Il est peu de domaines scientifiques et techniques dans lesquels autant de disciplines aussi variées travaillent en une si étroite collaboration. Une des caractéristiques de la radioprotection a été de réunir des personnes que normalement rien ne rapprochait et que souvent tout éloignait. Sans doute faut-il voir là d'une part l'étroite dépendance des problèmes traités par la radioprotection, d'autre part une grande partie de son développement depuis la dernière guerre dans des centres d'énergie nucléaire eux-mêmes multidisciplinaires. On peut dire que si la radioprotection est parvenue à un tel progrès, c'est pour avoir su réaliser l'intégration scientifique et la collaboration technique de toutes ces composantes. Ceci se traduit par la collaboration qui existe en matière de radioprotection entre les organismes nationaux et les organisations internationales.

Il était bien naturel qu'une conséquence secondaire de la multidisciplinarité de la radioprotection soit que ses réalisations profitent en retour aux différentes disciplines qu'elle avait sollicitées. Les études sur les suspensions, les aérosols et les poussières profitent à l'ensemble des études sur les pneumoconioses. En dosimétrie et surtout en analyse radioactive, les développements servent à la détection des impuretés ou des traces ainsi qu'à la mesure fine de nuisances chimiques, métalliques en particulier. En matière de prévention, les 
télémanipulateurs trouvent leur application immédiate dans la robotique, les enceintes de confinement, dans les domaines de l'élevage, de la pharmacie, de la bactériologie et de la médecine. Le système de limitation des expositions et les principes généraux de proction établis par la C.I.P.R. constituent une base pratique pour la lutte contre les autres nuisances. Aussi le principal avantage qu'en dehors de la radioprotection l'humanité puisse tirer de ces progrès, est d'en étendre l'application aux autres domaines de protection.

La radioprotection apparaît vraiment comme ayant un double mérite, le premier d'avoir su, en un quart de siècle, maîtriser pratiquement les problèmes posés par les nuisances radiologiques et radioactives, le second de servir d'exemple d'action contre les nuisances du monde moderne. 